\title{
Improving diagnosis of pediatric central nervous system tumours: aiming for early detection
}

\author{
Ran D. Goldman MD, Sylvia Cheng MD, D. Douglas Cochrane MD
}

- Cite as: CMAJ 2017 March 27;189:E459-63. doi: 10.1503/cmaj.160074

CMAJ podcasts: author interview at https://soundcloud.com/cmajpodcasts/160074-view

C entral nervous system (CNS) tumours account for a quarter of all cancers in children aged 1 to 19 years, despite being relatively uncommon. ${ }^{1}$ Survival in many cases is poor, and thus the diagnosis may be devastating. ${ }^{2}$ Yet for $70 \%$ of children with CNS tumours, there is potential for effective treatment with multimodal therapy by a specialized, multidisciplinary team. ${ }^{1}$ Early detection is essential to allow the best possible treatment by an experienced team of pediatric neurosurgeons and oncologists, to optimize outcomes. However, CNS tumours in children are often initially misdiagnosed as more common pediatric conditions, such as migraine, gastroenteritis, or psychologic or behavioural problems.

Headache, vomiting, lethargy and seizures are the most common symptoms associated with CNS tumours, but they are nonspecific and present more frequently with other, more benign conditions. The diagnosis of CNS tumours necessitates a comprehensive clinical history and complete examination, in addition to advanced imaging, such as computed tomography (CT) or magnetic resonance imaging (MRI) to confirm clinical suspicion. These imaging tests expose children to radiation or require preparation and sedation for young children; as such, clinicians should order these tests judiciously and only if merited. Here, we review the presenting features of and diagnostic approach to CNS tumours in children, to assist family physicians and pediatricians in the early identification of such lesions (Box 1).

\section{Which symptoms are associated with CNS tumours in childhood?}

Brain tumours have no unique pathognomonic features, which often makes early diagnosis difficult. ${ }^{3}$ Children commonly present with symptoms that may (but do not necessarily) reflect increased intracranial pressure. Headache and vomiting are two well-known symptoms that are associated with elevation of intracranial pressure, but that have frequently been attributed to more common conditions (e.g., headache to migraine and vomiting to gastrointestinal illness) or considered psychologic in origin. ${ }^{4}$ Many clinicians consider morning headache to be an important sign, caused by increased intracranial pressure after a long period of lying down. However, in one report, only two-thirds of children with CNS

\section{KEY POINTS}

- There are no pathognomonic features of brain tumours, making their diagnosis in children difficult.

- Headache, vomiting, lethargy and seizures are the most common symptoms associated with central nervous system tumours, but these occur at greater frequency with other, more benign conditions.

- Psychologic changes are frequent among children with central nervous system lesions, but they may be considered of psychosomatic origin.

- Despite advancement in imaging techniques and better access to computed tomography and magnetic resonance imaging, the prediagnostic symptomatic interval has remained virtually constant over the last several decades.

- The consequences of diagnostic delay can be devastating for patients and families.

tumours had headaches in the morning, ${ }^{5}$ and an absence of morning headaches does not rule out increased intracranial pressure due to space-occupying lesions in the brain. Furthermore, as many as $28 \%$ of children in one series had neither headaches nor vomiting, ${ }^{5}$ and in many instances, headaches are intermittent, fluctuating in severity during the day or over time. "Migraine headache" has been identified as a diagnosis associated with delayed diagnosis of CNS tumours, ${ }^{6}$ and reassessment must be considered for children with changes in migraine characteristics. ${ }^{6}$

In a Danish review of 46 consecutive pediatric admissions (over a period of 11 years) involving a CNS tumour, headache, vomiting and lethargy were the most frequent symptoms. ${ }^{7}$ In another Danish study, involving 55 children (median age at diagnosis 8.4 years), most of whom (59\%) had infratentorial tumours, vomiting (87\%), headache (focal in $69 \%$ of all children, awakening the child in $39 \%$ ), ataxia (35\%) and seizures (20\%) were the most common symptoms reported by patients. ${ }^{8}$ Among 60 children from two Japanese hospitals (median age at diagnosis 5.8 years), vomiting (24\%), headache $(17 \%)$, unsteadiness $(10 \%)$ and paresis $(10 \%)$ were the most common symptoms. ${ }^{9}$ In a French multicentre group, the most frequent signs among 166 patients were vomiting (72\%), psychomotor regression (60\%) and ataxia (60\%). ${ }^{5}$ 
In children with CNS tumours, a single sign or symptom, unless it is a prominent focal abnormality on neurologic examination, is uncommon at the time of diagnosis. In a series from Switzerland, in which headache, nausea or vomiting, seizures and behavioural changes were the most common symptoms, only $12 \%$ of patients had a single symptom, and a quarter of the children had seven or more signs and symptoms. ${ }^{3}$

Further frustrating early diagnosis is the absence of a single sequence of symptoms and signs that allows identification of CNS tumours in children. ${ }^{9}$ For example, although vomiting and headache are the most common symptoms, they can be the first sign, or they may occur with other signs or symptoms.

Psychologic changes were noted to be frequent among children with CNS tumours. Such changes may present an obstacle to timely diagnosis, because even when associated with somatic symptoms, they may be considered to be psychosomatic in origin. ${ }^{5}$ In two studies from the United Kingdom covering cases between the 1980s and 2001, a quarter of the 74 children in one series ${ }^{4}$ had diminished school performance, and half of the 274 children in the two series combined had behavioural changes - both findings leading to psychologic diagnoses and delay in identification of CNS tumour.,10

Age is an important factor affecting the presenting symptoms in children with CNS tumours. Among those younger than two years, the most common initial signs include seizures, vomiting, head tilt and behavioural changes, ${ }^{3}$ along with psychomotor regression. ${ }^{5}$ In one group of children under three years of age, drowsiness and anorexia were more frequent than among older children. ${ }^{5}$ In children older than two years, the most common initial symptoms include headache, vomiting, seizures, diplopia, ataxia and behavioural changes. ${ }^{3,5}$ History of impaired school performance, depression and anxiety are further important clues to the diagnosis. ${ }^{5}$

\section{What are the physical signs that suggest a CNS tumour?}

Most children with a CNS tumour will have notable signs on a full physical examination (Box 2). In an analysis of 3276 pediatric patients, less than $3 \%$ of those with headache and a brain tumour had no abnormality on neurologic examination, ${ }^{11}$ and among 72 children with brain tumours that started as headache, abnormalities on physical examination had developed in $85 \%$ within two months of headache onset. ${ }^{12}$ In one series, motor signs with paresis (17\%), an abnormal result on the finger-nose test (17\%) and

\section{Box 1: Evidence used in this review}

We used Canadian and US national guidelines to inform this review, in addition to published studies that were known to us. We identified additional articles through MEDLINE literature searches using various combinations of the search terms "pediatric," "brain tumours" and "children" from 1949 to the present. In addition, we reviewed conference abstracts and reference lists from seminal articles. Where possible, we selected the most recent articles and the articles with the most robust level of evidence (such as larger sample size, multicentre design or meta-analysis). We reviewed more than 100 citations generated by this search, of which 25 are included in this review. hyperreflexia (11\%) were the most common symptoms on examination. ${ }^{8}$ All other findings, including papilledema, were found in less than $10 \%$ of the 55 cases reviewed. ${ }^{8}$ A comprehensive physical examination, including funduscopy, is important to help providers identify deficits that should prompt imaging studies.

Although vomiting and headache are associated with every location of a CNS tumour in children, ${ }^{9}$ the site is frequently suggested by more specific symptoms. Infratentorial tumours present with ataxia (in about half of children with such tumours) ${ }^{8}$ and cranial nerve deficits, ${ }^{13}$ whereas supratentorial tumours present with seizures (in about a third of cases), ${ }^{8}$ motor and visual dysfunction, and endocrinologic abnormalities. ${ }^{13}$ Headache, vomiting and papilledema develop more rapidly in patients with infratentorial tumours, ${ }^{13}$ and hydrocephalus due to obstruction of outflow of the cerebrospinal fluid is more likely in these patients. Strabismus and facial and bulbar palsies were observed in children with brain stem tumours. ${ }^{9}$

Despite efforts to identify specific clinical patterns, variation in symptoms with location of the lesion and rate of tumour evolution results in patterns that are not consistently reliable. ${ }^{9}$ Figure 1 shows the tumour types and their respective locations that are most prevalent among infants, children and teenagers.

\section{What contributes to diagnostic delay?}

The prediagnostic symptomatic interval (PSI) can be subdivided into three components: PSI(1), the time from parents' identification of symptoms to first visit to a health care provider; PSI(2), the time from

Box 2: Findings of the physical examination in children with central nervous system tumours*
Cranial nerves
- Double vision
- Nystagmus
- Head tilt
- Facial palsy
- Abnormal eye movement
- Downward deviation of eyes
- Difficulty swallowing
- Deviation of tongue
- Reduced hearing
Other
- Paresis
- Dysmetria
- Hyperreflexia
- Papilledema
- Positive Romberg sign
- Increased muscle tone
- Hyporeflexia
- Clonus
- Decreased muscle tone
- Heel-knee-shin ataxia
*Based on common practice knowledge and authors' experience. ${ }^{8,9,11-13}$ 
first visit to specialist referral; and PSI(3), the time from referral until the diagnosis is made.

The time to diagnosis is critical in the effort to provide the best surgical and nonsurgical care for children. Despite a growing evidence base and improvements in the quality of and access to diagnostic imaging, there has been little improvement in the time to diagnosis of CNS tumours in children. Among 316 children in whom intracranial tumours were diagnosed over the period 1935 to 1959 , $50 \%$ had a total PSI of more than six months. ${ }^{14}$ Despite better access to CT in the 1990s, a study of 74 children $^{4}$ found a similar PSI of five months. Since then, the median for this interval has ranged from 7 to 230 days. $^{7-10,15-17}$
The authors of a study of 300 Israeli children, among whom the average total PSI was 7.7 (standard deviation 16.7) months, concluded that about a quarter of the children could have had an earlier diagnosis and that diagnostic delay was associated with delayed referral to imaging. ${ }^{18}$ Only half of the children in this cohort had deterioration of symptoms after first presentation. Torticollis, ataxia and motor dysfunction were the most common symptoms in those whose diagnosis was delayed. ${ }^{18}$

In a series from Denmark, the median duration of PSI(1) was seven days (with a wide range, 0-365 d), of PSI(2) was nine days (range 0-730 d), of PSI(3) was six days (range 0-231 d) and of total PSI was 66 days (range $0-730 \mathrm{~d}$ ). ${ }^{8}$ In another Danish series of 46 chil-

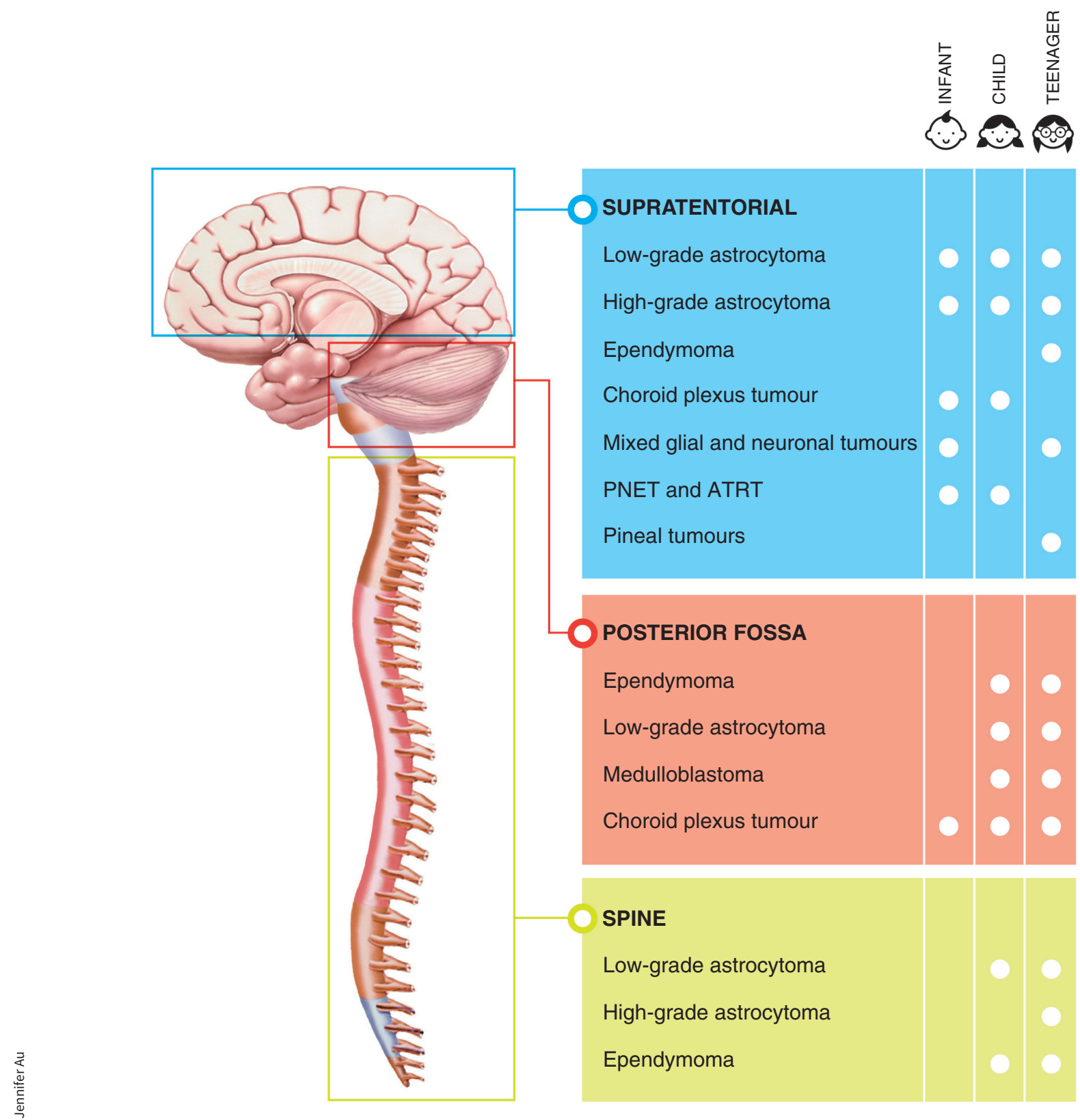

Figure 1: Pediatric brain tumour types and respective locations that are most prevalent among infants, children and teenagers. ATRT = atypical teratoid rhabdoid tumour, PNET = primitive neuroectodermal tumour. 
dren seen in a hospital pediatric department, $80 \%$ had a diagnosis within four days of admission, and another $10 \%$ after more than one week. ${ }^{7}$ In Japan, where there is better access to neuroimaging, the median total PSI was 20.5 days (excluding diagnosis at regular check-ups and prenatal diagnosis), and there was no significant difference in median PSI among patients with four common initial symptoms (headache, vomiting, unsteadiness, paresis) or any neurologic signs. ${ }^{9}$ In a Swiss report, PSI(1) was 14 days (range 0-2310 d) and PSI(2) was 30 days; the median total PSI of 60 days (range 0-3010 d) was considered long. ${ }^{3}$ The authors of a German study of 245 patients seen between 1980 and 2004 reported a total PSI of 24 days, ${ }^{16}$ with shorter interval to diagnosis associated with motor and eye dysfunction, focal disorders, early morning vomiting and disturbed alertness, whereas the median PSI in a UK study (1988 to 2001) was 75 days (range $1 \mathrm{~d}$ to $120 \mathrm{mo}){ }^{4}$

A short symptomatic interval commonly reflects more rapid progression and was significantly correlated with high-grade tumours and age three years or younger. ${ }^{15}$ In Canada's Atlantic provinces, mean PSI was as long as 7.3 (95\% confidence interval 4.99-9.67) months, and less than half $(41 \%)$ of cases were diagnosed within three physician visits. ${ }^{15}$ Medulloblastoma was associated with a shorter PSI, and brain stem tumours took longer to identify. ${ }^{15}$

Several factors affect the PSI, including symptoms and age. Median PSI durations of 30 (range 3-330) days with vomiting, 75 (range 5-730) days with headache and 75 (range 1-730) days with ataxia were reported in one series of 55 children. ${ }^{8}$ In 166 children with medulloblastoma in France, the presence of psychologic symptoms was associated with a significantly longer PSI relative to other types of symptoms ( $91 \mathrm{v} .60 \mathrm{~d}, p=0.001$ )..$^{5}$ Older children had longer PSI (145 v. $57 \mathrm{~d}, p<0.0001) .{ }^{5}$ Prolonged PSI was noted when headaches and vomiting were inconsistent or presented late in the course, if symptoms spontaneously resolved and among those whose initial symptoms were psychologic. ${ }^{5}$

Infants are reported to have a shorter PSI than older children, ${ }^{3,5}$ possibly because of closer observation by parents and health care providers, the invasive nature of tumours in early infancy and the higher rate of symptoms due to increased intracranial pressure.

Preadmission delay also depends on tumour grade and anatomic location,,$^{3,7,9,17}$ with brain stem tumours diagnosed after 10 days, those in the posterior fossa after 20.5 days ${ }^{9,17}$ and others after 30 days (differences not statistically significant). ${ }^{9}$ The PSI for children with medulloblastoma was longer, at 65 days. ${ }^{5}$ Ease of access to advanced imaging depends on geographic location. In countries such as Japan and the United States, access to CT and MRI is easy and can be arranged quickly, even in relatively small centres. In Canada, CT and MRI are much less accessible. ${ }^{19}$

\section{What are the consequences of diagnostic delay?}

A delay in diagnosis of CNS tumours in children can be devastating for both families and clinicians. Delayed diagnosis may also lead to anxiety and lack of trust in the health care system on the part of patients and families. ${ }^{20}$ However, it often takes time for patients, families and physicians to recognize that a child's condi- tion is abnormal. Considering symptoms in retrospect, with hindsight bias (the "saw-it-all-along" effect), frequently leads to harsh judgment of assessment and management decisions made at the time of initial complaints. ${ }^{21}$

Time to diagnosis affects time to treatment, and delays may lead to neurologic, developmental and neuroendocrinologic morbidity, such as loss of visual pathway or hypothalamic pituitary axis with supratentorial tumours, as well as substantial long-term deficits involving physical, sensory, cognitive, neurologic and endocrine complications. ${ }^{1}$ Delays have also been associated with impaired intellectual function. ${ }^{22,23}$ More prospective studies are needed to determine the impact of diagnostic and treatment delays on survival.

\section{What knowledge translation strategies can help to ensure timely diagnosis?}

In an effort to avoid routine neuroimaging studies for children with recurrent headaches and normal results on neurologic examination, ${ }^{24}$ organizations worldwide are looking for strategies to increase patient involvement, to ensure responsive primary and community care, and to change models of care as needed. ${ }^{4}$ One initiative to enhance the public's and health care providers' awareness of symptoms is the UK-based HeadSmart website, which offers detailed description of symptoms. ${ }^{25}$ Also in the UK, the National Institute for Health and Clinical Excellence offers guidance aimed at standardizing services for children and young people with cancer. ${ }^{26}$

A consensus statement derived by 120 health care providers and parents ${ }^{6}$ presented 64 recommendations related to timely diagnosis. Initial management should include imaging after four weeks of persistent headache, after four weeks of persistent lethargy or withdrawal (in preschool children), after two weeks of persistent nausea or vomiting (or both), or after two weeks of persistent visual changes. Patients should be referred to a pediatrician or a pediatric neurologist if symptoms persist or if physical examination suggests any of the signs and symptoms listed in Box 2.

\section{Unanswered questions}

Several important questions remain unanswered. The acceptable time between first detection of symptoms and imaging must be determined, to balance the risk associated with imaging against the benefit of earlier detection of CNS tumours. It would be helpful to develop a decision rule to determine the type and timing of investigation and follow-up for children with common symptoms such as headache and vomiting. Finally, effective knowledge translation must be designed for parents, to allow them to seek medical attention as soon as symptoms suggestive of CNS tumours arise.

\section{Conclusion}

Physicians should consider CNS tumours in the differential diagnosis for children with headache, vomiting, lethargy, ataxia, visual changes, prolonged torticollis, nystagmus, papilledema or sei- 
zures, and should consider appropriate imaging and specialist referral according to the history and a comprehensive physical examination. For patients with nonspecific symptoms and normal results on neurologic examination, imaging should be considered if the symptoms do not resolve as expected. Standards for acceptable prediagnostic symptomatic intervals must be established to avoid delay in diagnosis and suboptimal outcomes.

For patients with paresis or unsteadiness, prompt imaging should be strongly considered. For those with headache or vomiting and normal results on a detailed neurologic examination, imaging should be considered if symptoms do not resolve after several weeks.

\section{References}

1. Armstrong GT. Long-term survivors of childhood central nervous system malignancies: the experience of the Childhood Cancer Survivor Study. Eur J Paediatr Neurol 2010;14:298-303.

2. Sankila R, Martos Jiménez MC, Miljus D, et al. Geographical comparison of cancer survival in European children (1988-1997): report from the Automated Childhood Cancer Information System project. Eur J Cancer 2006;42:1972-80.

3. Dobrovoljac M, Hengartner H, Boltshauser E, et al. Delay in the diagnosis of paediatric brain tumours. Eur J Pediatr 2002;161:663-7.

4. Delayed diagnosis of cancer: thematic review. Redditch (UK): National Patient Safety Agency; 2010. Available: www.nrls.npsa.nhs.uk/EasySiteWeb/getresource .axd?AssetID=69895\& (accessed 2016 Jan. 14).

5. Brasme JF, Chalumeau M, Doz F, et al. Interval between onset of symptoms and diagnosis of medulloblastoma in children: distribution and determinants in a population-based study. Eur J Pediatr 2012;171:25-32.

6. Wilne $\mathrm{S}$, Koller K, Collier J, et al. The diagnosis of brain tumours in children: a guideline to assist healthcare professionals in the assessment of children who may have a brain tumour. Arch Dis Child 2010;95:534-9.

7. Løhmann DJ, Sørensen P, Jørgensen J, et al. Most central nervous system tumours in children are diagnosed with little delay after admission. Dan Med J 2014; 61:A4886.

8. Klitbo DM, Nielsen R, Illum NO, et al. Symptoms and time to diagnosis in children with brain tumours. Dan Med Bull 2011;58:A4285.

9. Hayashi N, Kidokoro H, Miyajima Y, et al. How do the clinical features of brain tumours in childhood progress before diagnosis? Brain Dev 2010;32:636-41.

10. Wilne SH, Ferris RC, Nathwani A, et al. The presenting features of brain tumors: a review of 200 cases. Arch Dis Child 2006;91:502-6.

11. Childhood Brain Tumor Consortium. The epidemiology of headache among children with brain tumor. J Neurooncol 1991;10:31-46.

12. Honig PJ, Charney EB. Children with brain tumor headaches. Distinguishing features. Am J Dis Child 1982;136:121-4.

13. Wilne S, Collier J, Kennedy C, et al. Presentation of childhood CNS tumours: a systematic review and meta-analysis. Lancet Oncol 2007;8:685-95.

14. Gjerris F. Clinical aspects and long-term prognosis of intracranial tumors in infancy and childhood. Dev Med Child Neurol 1976;18:145-59.

15. Mehta V, Chapman A, McNeely PD, et al. Latency between symptom onset and diagnosis of pediatric brain tumors: an eastern Canadian geographic study. Neurosurgery 2002;51:365-72.
16. Reulecke BC, Erker CG, Fiedler BJ, et al. Brain tumors in children: initial symptoms and their influence on the time span between symptom onset and diagnosis. J Child Neurol 2008;23:178-83.

17. Duffner PK. Diagnosis of brain tumours in children. Expert Rev Neurother 2007; 7:875-85.

18. Shay V, Fattal-Valevski A, Beni-Adani L, et al. Diagnostic delay of pediatric brain tumors in Israel: a retrospective risk factor analysis. Childs Nerv Syst 2012;28: 93-100.

19. Emery DJ, Forster AJ, Shojania KG, et al. Management of MRI wait lists in Canada. Healthc Policy 2009;4:76-86.

20. Mazor KM, Roblin DW, Greene SM, et al. Toward patient-centered cancer care: patient perceptions of problematic events, impact, and response. J Clin Oncol 2012; 30:1784-90.

21. Sanna LJ, Schwarz N, Small EM. Accessibility experiences and the hindsight bias: I knew it all along versus it could never have happened. Mem Cognit 2002;30:1288-96.

22. de Ruiter MA, Van Mourik R, Schouten-van Meeteren AY, et al. Neurocognitive consequences of a paediatric brain tumour and its treatment: a meta-analysis. Dev Med Child Neurol 2013;55:408-17.

23. Lassaletta A, Bouffet $E$, Mabbott $D$, et al. Functional and neuropsychological late outcomes in posterior fossa tumors in children. Childs Nerv Syst 2015; 31:1877-90.

24. Lewis DW, Ashwal S, Dahl G, et al.; Quality Standards Subcommittee of American Academy of Neurology; Practice Committee of Child Neurology Society. Practice parameter: evaluation of children and adolescents with recurrent headaches: report of the Quality Standards Subcommittee of the American Academy of Neurology and the Practice Committee of the Child Neurology Society. Neurology 2002;59:490-8.

25. HeadSmart Be Brain Tumour Aware. A new clinical guideline from the Royal College of Paediatrics and Child Health with a national awareness campaign accelerates brain tumor diagnosis in UK children - "HeadSmart: Be Brain Tumour Aware." Neuro Oncol 2016;18:445-54.

26. National Collaborating Centre for Cancer. Guidance on cancer services: improving outcomes in children and young people with cancer. The evidence review. London (UK): National Institute for Health and Clinical Excellence; 2005. Available: www.nice. org.uk/guidance/CSG7/documents/improving-outcomes-in-children-and-young -people-with-cancer-evidence-review-2 (accessed 2016 Nov. 5).

\section{Resources for physicians and families}

- Agency for Healthcare Research and Quality (US): www.guideline .gov/content.aspx?id=49094

- National Cancer Institute (US): www.cancer.gov/types/brain/hp/ child-brain-treatment-pdq

- Children's Oncology Group: www.survivorshipguidelines.org/

- National Patient Safety Agency (UK): www.nrls.npsa.nhs.uk/ EasySiteWeb/getresource.axd?AssetID=69895\&

- HeadSmart project (UK): www.headsmart.org.uk/

- Pediatric Oncology Group of Ontario: www.pogo.ca/healthcare/ guidelinedocuments/

\section{Competing interests: None declared.}

This article has been peer reviewed.

Affiliations: Pediatric Research in Emergency Therapeutics (PRETx) Program, Department of Pediatrics (Goldman), Division of Hematology, Oncology and Bone Marrow Transplant, Depart- ment of Pediatrics (Cheng), and Division of Pediatric Neurosurgery, Department of Surgery (Cochrane), BC Children's Hospital, Vancouver, BC; Child and Family Research Institute (Goldman, Cheng, Cochrane), University of British Columbia, Vancouver, BC
Contributors: All of the authors wrote the article and revised it for important intellectual content. All of the authors approved the version to be published and agreed to act as guarantors of the work.

Correspondence to: Ran Goldman, rgoldman@cw.bc.ca 